\title{
Structural insight into mechanisms for dynamic regulation of PKM2
}

\author{
Ping Wang ${ }^{1}$, Chang Sun ${ }^{1}$, Tingting $\mathrm{Zhu}^{1}$, Yanhui $\mathrm{Xu}^{1,2 \bowtie}$ \\ ${ }^{1}$ Fudan University Shanghai Cancer Center and Institute of Biomedical Sciences, Shanghai Medical College of Fudan \\ University, Shanghai 200032, China \\ 2 State Key Laboratory of Genetic Engineering, School of Life Sciences, Fudan University, Shanghai 200433, China \\ $\triangle$ Correspondence: xuyh@fudan.edu.cn (Y. Xu)
}

Received December 23, 2014 Accepted December 26, 2014

\begin{abstract}
Pyruvate kinase isoform M2 (PKM2) converts phosphoenolpyruvate (PEP) to pyruvate and plays an important role in cancer metabolism. Here, we show that posttranslational modifications and a patient-derived mutation regulate pyruvate kinase activity of PKM2 through modulating the conformation of the PKM2 tetramer. We determined crystal structures of human PKM2 mutants and proposed a "seesaw" model to illustrate conformational changes between an inactive T-state and an active R-state tetramers of PKM2. Biochemical and structural analyses demonstrate that $\mathrm{PKM} 2^{\mathrm{Y} 105 \mathrm{E}}$ (phosphorylation mimic of Y105) decreases pyruvate kinase activity by inhibiting FBP (fructose 1,6-bisphosphate)induced R-state formation, and PKM2 ${ }^{\mathrm{K} 305 \mathrm{Q}}$ (acetylation mimic of K305) abolishes the activity by hindering tetramer formation. K422R, a patient-derived mutation of PKM2, favors a stable, inactive T-state tetramer because of strong intermolecular interactions. Our study reveals the mechanism for dynamic regulation of PKM2 by posttranslational modifications and a patient-derived mutation and provides a structural basis for further investigation of other modifications and mutations of PKM2 yet to be discovered.
\end{abstract}

KEYWORDS pyruvate kinase M2, crystal structure, allosteric regulation, Warburg effect, post-translational modifications

Ping Wang and Chang Sun have contributed equally to this work.

Electronic supplementary material The online version of this article (doi:10.1007/s13238-015-0132-x) contains supplementary material, which is available to authorized users.

\section{INTRODUCTION}

Most somatic cells produce energy predominantly through oxidative phosphorylation, whereas cancer cells produce energy mainly through the less efficient glycolytic pathway, followed by lactic acid production under aerobic conditions (Warburg, 1956). This aerobic glycolysis (also known as the Warburg effect) plays an important role in tumorigenesis (Vander Heiden et al., 2009; Koppenol et al., 2011; Chaneton and Gottlieb, 2012; Yang and Lu, 2013; Wong et al., 2015). Pyruvate kinase transfers one phosphate group from phosphoenolpyruvate (PEP) to adenosine di-phosphate (ADP), and thus generates one pyruvate molecule and one adenosine tri-phosphate (ATP) molecule. This step is one of the rate-limiting steps of glycolysis, and therefore pyruvate kinase is one of the primary mediators of glycolysis.

Four mammalian isoforms of pyruvate kinase-PKL, PKR, PKM1 and PKM2-are expressed in various types of cells and tissues. PKL is expressed in the liver, and PKR is expressed in the red blood cells. PKM1 and PKM2 are derived from alternative splicing of the PKM gene (Noguchi et al., 1986; Noguchi et al., 1987). PKM1 is a constitutively active isoform expressed in differentiated cells from various tissues. In contrast, PKM2 has low basal activity and is activated by an effector molecule fructose 1,6-bisphosphate (FBP). PKM2 is expressed in most proliferating cells (Christofk et al., 2008a). Furthermore, the regulation of PKM2 pyruvate kinase activity plays an essential role in cancer metabolism and is crucial for the growth and survival of cancer cells (Chaneton and Gottlieb, 2012; Yang and Lu, 2013; Wong et al., 2015). Recently, PKM2 has been reported to function as a nuclear protein kinase to regulate gene transcription and promote tumorigenesis (Gao et al., 2012; Yang et al., 2012a, b; Gao et al., 2013; Lv et al., 2013; Keller et al., 2014).

PKM2 exists in a dynamic population of monomer, dimer and tetramer and its pyruvate kinase activity relies on the 
formation of the tetramer. Upon formation of the tetramer, PKM2 can adopt the inactive T-state or active R-state conformation (Morgan et al., 2013). The pyruvate kinase activity of PKM2 is regulated by metabolic intermediates and posttranslational modifications. For example, metabolic intermediates such as FBP and $\mathrm{N}$-succinyl-5-aminoimidazole-4carboxamide ribose-5'-phosphate (SAICAR) increase the pyruvate kinase activity of PKM2 (Dombrauckas et al., 2005; Chaneton et al., 2012; Keller et al., 2012). Synthetic compounds have been screened for their ability to activate PKM2 and, thus, suppress cancer growth (Boxer et al., 2010; Jiang et al., 2010; Anastasiou et al., 2012; Kung et al., 2012). Some of these compounds have been shown to promote tetramer formation of PKM2 and enhance its enzymatic activity (Anastasiou et al., 2012). In addition, serine and phenylalanine have been reported to bind to the allosteric site of PKM2 and activate or inhibit its activity, respectively (Chaneton et al., 2012; Morgan et al., 2013).

Post-translational modifications including phosphorylation, oxidation, hydroxylation and acetylation also regulate PKM2 activity to promote cancer proliferation. For example, acetylation of residue K305 inhibits pyruvate kinase activity of PKM2 in vitro (Lv et al., 2011) and acetylation of residue K433 affects FBP binding and prevents PKM2 activation (Lv et al., 2013). Phosphorylation of residue Y105 inhibits the tetramer formation and pyruvate kinase activity of PKM2 (Hitosugi et al., 2009). Furthermore, oxidation of residue C358 inhibits PKM2 activity and promotes the metabolic changes required for proliferation (Anastasiou et al., 2011). PKM2 hydroxylation of P403 and P408 promotes HIF-1 transactivation in cancer cells (Luo et al., 2011). The mutation R399E of PKM2 (PKM2 ${ }^{\mathrm{R} 399 \mathrm{E}}$ ) was shown to disrupt the tetramer formation on one of dimer interfaces, thereby producing dimers and decreasing its pyruvate kinase activity (Gao et al., 2012). In addition, mutations K422R and H391Y of PKM2 (PKM2 ${ }^{\mathrm{K} 422 \mathrm{R}}$ and $\mathrm{PKM} 2^{\mathrm{H} 391 \mathrm{Y}}$ ) were shown to decrease its pyruvate kinase activity in Bloom Syndrome (BS) patients, who are prone to cancer (Anitha et al., 2004; Akhtar et al., 2009; Gupta et al., 2010; lqbal et al., 2014).

Although PKM2 has been studied for decades, how its activity is regulated remains poorly understood. In this study, we revealed how pyruvate kinase activity of $\mathrm{PKM} 2$ is regulated by post-translational modifications and a patientderived mutation. On the basis of our observations, we propose a model for dynamic regulation of PKM2. Our study also provides a structural basis for further investigation of dynamic regulation of PKM2 by other post-translational modifications and mutations involved in cancer metabolism.

\section{RESULTS}

Effects of post-translational modifications and a patient-derived mutation on PKM2 activity

To investigate how the enzymatic activity of PKM2 is regulated, we purified wild-type PKM2 (PKM2 $\left.{ }^{\mathrm{WT}}\right), \mathrm{PKM} 2^{\mathrm{R} 399 \mathrm{E}}(\mathrm{a}$ dimeric mutant) (Gao et al., 2012) and PKM2 ${ }^{\mathrm{K} 422 \mathrm{R}}$ (a patientderived mutation). Given the difficulty to obtain phosphorylated or acetylated PKM2 proteins, we purified PKM2 ${ }^{\mathrm{Y} 105 \mathrm{E}}$ (a phosphorylation mimic of $\mathrm{Y} 105$ ) and $\mathrm{PKM} 2^{\mathrm{K} 305 \mathrm{Q}}$ (an acetylation mimic of $\mathrm{K} 305$ ) to mimic PKM2 containing the two modifications. The above four PKM2 mutants and PKM2 ${ }^{\mathrm{WT}}$ were used for enzymatic activity assays and structural studies (Fig. S1A). We first measured the enzymatic activities for wild-type and mutants of PKM2 and calculated the $\mathrm{Km}$ values for PEP. The kinetic activities were calculated based on the PEP saturation curves in the absence or presence of FBP, an allosteric activator of PKM2 (Fig. 1A and 1B). We also calculated the normalized pyruvate kinase activity $\left(\mathrm{kcat} / \mathrm{Km},(\mathrm{mol} / \mathrm{L})^{-1} \mathrm{~s}^{-1}\right)$, as represented by the value of $\mathrm{Vmax} / \mathrm{Km}$ of $\mathrm{PKM} 2^{\mathrm{WT}}$, because an equal amount of protein was used for all activity assays (Fig. 1C). We could not detect protein kinase activity for human PKM2 under our experimental conditions using histone $\mathrm{H} 3$ as substrate (data not shown). Therefore, only pyruvate kinase activities (enzymatic activity hereafter if not specified) were measured for wild-type and mutants of PKM2. A high enzyme concentration in the reaction would greatly decrease the accuracy of calculated reaction rate. Because of the extremely fast reaction rate (less than $1.5 \mathrm{~min}$ ), we measured the pyruvate kinase activity in nanomolar concentrations.

In the presence of FBP, PKM2 ${ }^{\mathrm{WT}}$ showed a significant increase in enzymatic activity (compared to that in the absence of FBP) with a normalized $\mathrm{kcat} / \mathrm{Km}$ of 5.6 and a decrease in $\mathrm{Km}$ to $0.2 \mathrm{mM}$ (Fig. $1 \mathrm{~A}-\mathrm{C}$ and Table S1). $\mathrm{PKM} 2^{\mathrm{K} 305 \mathrm{Q}}$ showed barely detectable pyruvate kinase activity in the absence of FBP, and a normalized kcat $/ \mathrm{Km}$ of 0.2 in the presence of FBP, indicating a significant decrease in activity. Compared to $\mathrm{PKM} 2^{\mathrm{WT}}, \mathrm{PKM} 2^{\mathrm{R} 399 \mathrm{E}}$ showed a decrease in activity in the absence or presence of FBP, with a $\mathrm{kcat} / \mathrm{Km}$ of 0.6 or 1.8 , respectively. Interestingly, $\mathrm{PKM} 2^{\mathrm{Y} 105 \mathrm{E}}$ showed a slight decrease in activity in the presence, but not the absence, of FBP, with a kcat $/ \mathrm{Km}$ of 1.2 in the absence of FBP and 4.3 in the presence of FBP. In contrast, $\mathrm{PKM} 2^{\mathrm{K} 422 \mathrm{R}}$ significantly decreased the enzymatic activity (kcat/Km of 0.2 ) and substrate binding affinity only in the absence of FBP. Taken together, these mutations showed different effects on pyruvate kinase activity of PKM2.

\section{Enzymatic activities of PKM2 mutants are not positively} correlated with PKM2 tetramer formation

Tetramer formation is essential for the pyruvate kinase activity of PKM2 (Dombrauckas et al., 2005). The gel-filtration analyses of wild-type and mutants of PKM2 show a mixed population of PKM2 in monomer, dimer and tetramer (Figs. 1D, 1E and S1B). PKM2 ${ }^{\mathrm{WT}}$ appears to preferentially form a dimer in the absence of FBP and tends to form a tetramer in the presence of FBP. Compared to $\mathrm{PKM} 2^{\mathrm{WT}}$, $\mathrm{PKM} 2^{\mathrm{Y} 105 \mathrm{E}}$ and $\mathrm{PKM} 2^{\mathrm{R} 399 \mathrm{E}}$ showed similar patterns of oligomerization in the absence of FBP. In contrast, 



Figure 1. Activities and tetramer formation for wild-type PKM2 and mutants of PKM2. (A and B) Kinetic activities of PKM2 proteins in the absence $(A)$ or presence $(B)$ of FBP. The data were fit with the Allosteric Sigmoidal Equation $(A)$ or the MichaelisMenten Equation (B). (C) Normalized activities ( $\mathrm{kcat} / \mathrm{Km}$ ) of wild-type PKM2 and mutants of PKM2 calculated according to the results from Fig. $1 \mathrm{~A}$ and $1 \mathrm{~B}$ with the value $(\mathrm{kcat} / \mathrm{Km})$ of $\mathrm{PKM} 2^{\mathrm{WT}}$ as a standard. The error bars represent mean \pm SD for triplicate experiments. (D and E). Gel filtration of wild-type and mutants of PKM2 in the absence (D) or presence (E) of FBP. Peak positions of monomer, dimer and tetramer are indicated as dashed lines. The color scheme for PKM2 proteins is indicated. Superdex 200 (GE Healthcare, 10/300 GL) was used in gel-filtration analyses.

$\mathrm{PKM} 2^{\mathrm{K} 305 \mathrm{Q}}$ tended to form a monomer whereas $\mathrm{PKM} 2^{\mathrm{K} 422 \mathrm{R}}$ mainly formed a tetramer in the absence of FBP. In the presence of $\mathrm{FBP}, \mathrm{PKM} 2^{\mathrm{Y} 105 \mathrm{E}}$ and $\mathrm{PKM} 2^{\mathrm{R} 399 \mathrm{E}}$ only slightly increased their tendency to form a tetramer, whereas $\mathrm{PKM} 2^{\mathrm{K} 305 \mathrm{Q}}$ only formed a dimer; $\mathrm{PKM} 2^{\mathrm{K} 422 \mathrm{R}}$ maintained its tendency to form the tetramer. The above observations are generally consistent with the prediction that the more tetramer that forms, the more active the protein is. However, exceptions exist. For example, in the absence of FBP, $\mathrm{PKM} 2^{\mathrm{K} 422 \mathrm{R}}$ showed a significantly high level of tetramer formation, but was much less active than PKM2 ${ }^{\mathrm{WT}}$. The results indicate that tetramer formation alone cannot explain how PKM2 activity is regulated by these mutations.

It was also noted that PKM2 ${ }^{\mathrm{WT}}$ formed a tetramer in a concentration- and time-dependent manner (Fig. S1C and S1D). As indicated by size exclusive chromatography, the higher concentration of protein used, the more tetramer formed (Fig. S1C). Interestingly, monomeric PKM2 eluted when either tetrameric or monomeric PKM2 was re-subjected to size exclusion chromatography under similar experimental conditions (Fig. S1D). To minimize concentration- and time-dependent confounding factors, we compared the oligomerization states of PKM2 ${ }^{\mathrm{WT}}$ and all the mutants under similar protein concentrations and experimental conditions.

\section{Enzymatic activities of PKM2 mutants are not positively correlated with their thermal stability}

The thermal stability of PKM2 was reported to reflect its enzymatic activity (Morgan et al., 2010). Thus, we next performed a thermal-shift assay to investigate the correlation 
between enzymatic activity and thermal stability of PKM2 proteins. PKM2 ${ }^{\mathrm{WT}}$ and $\mathrm{PKM} 2^{\mathrm{Y} 105 \mathrm{E}}$ showed two peaks (Tm: $\sim 44^{\circ} \mathrm{C}$ and $\sim 60^{\circ} \mathrm{C}$ ) in the absence of FBP and a single peak ( Tm value of $\sim 59^{\circ} \mathrm{C}$ ) in the presence of FBP (Fig. S1E, Table $\mathrm{S} 1)$. Compared to $\mathrm{PKM} 2^{\mathrm{WT}}, \mathrm{PKM} 2^{\mathrm{K} 305 \mathrm{Q}}$ was less stable in the absence $\left(\mathrm{Tm}: \sim 44^{\circ} \mathrm{C}\right)$ and presence $\left(\mathrm{Tm}: \sim 54^{\circ} \mathrm{C}\right)$ of FBP, which is consistent with its lesser tendency toward tetramer formation and lower enzymatic activity. The $\mathrm{Tm}$ values of $\mathrm{PKM} 2^{\mathrm{R} 399 \mathrm{E}}\left(\sim 56^{\circ} \mathrm{C}\right)$ and $\mathrm{PKM} 2^{\mathrm{K} 422 \mathrm{R}}\left(\sim 61^{\circ} \mathrm{C}\right)$ were slightly enhanced by the addition of FBP, indicating that both proteins are relatively stable and FBP could not significantly enhance their thermal stabilities. These results indicate that thermal stability is not positively correlated with enzymatic activity or tetramer formation of the wild-type and mutants of PKM2 tested in this study. Taken together, the above results indicate that changes of the pyruvate kinase activity of PKM2 could not be predicted based on the state of oligomerization or thermal stability.

\section{Crystal structures of human PKM2 mutants}

To investigate the molecular mechanism for the regulation of PKM2 activity, we determined the crystal structures of $\mathrm{PKM} 2^{\mathrm{K} 305 \mathrm{Q}}, \mathrm{PKM} 2^{\mathrm{Y} 105 \mathrm{E}}, \mathrm{PKM} 2^{\mathrm{R} 399 \mathrm{E}}$ and $\mathrm{PKM} 2^{\mathrm{K} 422 \mathrm{R}}$ in the absence of $F B P$, in addition to $P K M 2^{K 422 R}$ in the presence of FBP (PKM2 ${ }^{\mathrm{K} 422 \mathrm{R} \_\mathrm{FBP}}$ ) (Fig. S2A and Table 1). Although $\mathrm{PKM}^{\mathrm{WT}}$ and PKM2 mutants (except $\mathrm{PKM} 2^{\mathrm{K} 422 \mathrm{R}}$ ) were present in the form of a monomer in a certain percentage in solution with low protein concentration (less than $0.2 \mathrm{mg} / \mathrm{mL}$ ) (Fig. 1D), all PKM2 proteins formed tetramer in the crystals. The results are consistent with the observation that higher protein concentration leads to more tetramer formation for PKM2 proteins in gel filtration (Fig. S1C). The crystal structures of wild-type PKM2 in the presence of oxalate (PKM2 ${ }^{\text {Oxalate) }}$ (PDB: 3BJT) (Christofk et al., 2008b) and $\mathrm{PKM} 2$ in complex with phenylalanine (PKM2 ${ }^{\mathrm{Phe}}$ ) (PDB: 4FXJ) (Morgan et al., 2013) were used for the following structural comparison. In addition, we drafted a model for four monomers in the tetramer structure to clarify the following description (Fig. S2A).

The first striking observation from the structural analyses is that $\mathrm{PKM} 2^{\mathrm{Oxalate}}, \mathrm{PKM} 2^{\mathrm{K} 305 \mathrm{Q}}$ and $\mathrm{PKM} 2^{\mathrm{K} 422 \mathrm{R} \text { FBP }}$ adopted the R-state conformation, whereas all other PKM2 mutants adopted the T-state conformation (Fig. S2A). Notably, phenylalanine was previously reported to function as an inhibitor to lock PKM2 in an inactive T-state conformation (Morgan et al., 2013). PKM2 Oxalate adopted an R-state conformation because the crystals were obtained in the presence of oxalate (an analog of PEP), which may induce an R-state conformation of PKM2 (Christofk et al., 2008b). Similarly, PKM2 $2^{\mathrm{K} 305 \mathrm{Q}}$ adopted an R-state conformation because the protein was crystallized in the presence of malonate $(0.2 \mathrm{~mol} / \mathrm{L})$, which was observed in the active site of the PKM $2^{\mathrm{K} 305 \mathrm{Q}}$ structure (Fig. S2B). Both oxalate and malonate are analogs of PEP and may favor the formation of the R-state tetramer. These results suggest that wild-type
PKM2 and mutants of the PKM2 tetramer tend to adopt the T-state conformation in the apo form but prefer an R-state conformation in the presence of either FBP or an analog of PEP.

\section{A "seesaw" model in the R-/T-state transition of PKM2}

Previous studies have demonstrated that a PKM2 tetramer in the R-state (active) is more active than that in the T-state (inactive). Structural comparison indicates that each individual monomer of PKM2 adopts similar fold with a rootmean-squared deviation (rmsd) of less than $0.6 \AA$ for approximately 430 aligned $\mathrm{C} \alpha$ atoms (Fig. S2C). However, structure analysis indicates that the PKM2 tetramer undergoes significant changes between $\mathrm{R}$ - and T-state conformations with a rotation about the helix $\alpha 9$ of five degrees for each monomer (Fig. 2B and 2C). This observation is consistent with previous studies of the PKM protein in Leishmania Mexicana (Morgan et al., 2010). The PKM2 tetramer is formed through intermolecular interactions between four monomers on large $\left(A-A^{\prime}\right)$ and small $\left(C-C^{\prime}\right)$ interfaces (Fig. 2A). We propose a seesaw model for overall conformational changes during transitions between the $\mathrm{R}$-state $\left(\mathrm{PKM} 2^{\mathrm{K} 305 \mathrm{Q}}\right)$ and the T-state (PKM2 $\left.{ }^{\mathrm{K} 422 \mathrm{R}}\right)$ (Fig. 2B and $\left.2 \mathrm{C}\right)$.

On the $\mathrm{C}-\mathrm{C}^{\prime}$ interface, each monomeric PKM2 adopts a rigid conformation and rotates alone with strand $\beta 20$ (Fig. 2B-D). When PKM2 adopts the T-state conformation, the FBP activation motif (FAM, residues G514-T522) and helix $\alpha 18$ on monomer $A$ interact with FAM and $\alpha 18$ on monomer $B$ on one side of two parallel seesaws. Helices a14/a15 on monomer $A$ and $\alpha 14 / \alpha 15$ on monomer $B$ are apart from one another on the other side (Figs. 2D and S3). In a process of transitioning from the T-state to the R-state, FAM/ 18 on monomer $A$ and FAM/a18 on monomer B move away from one another, and helices a14/a15 on monomer $A$ and $\alpha 14 / \alpha 15$ on monomer $B$ move closer to one another. Consistent with the above observations, the distance between residues M525 ( $\beta 20)$ on monomer A and M525 $(\beta 20)$ on monomer $B$ is approximately $5.0 \AA$ in either the Ror T-state tetramer (Table S2). In contrast, the distance between residues $L 488$ (in a18) on monomer $A$ and $L 488$ (in a18) on monomer $B$ is $\sim 25.9 \AA$ in the T-state and $\sim 27.4 \AA$ in the R-state conformation, respectively; the distance between residues $E 397$ (in a14) on monomer $A$ and $E 397$ (in a14) on monomer $B$ is $\sim 20.7 \AA$ in T-state and $\sim 14.0 \AA$ in the R-state conformation.

On the $A-A^{\prime}$ interface, each monomeric PKM2 rotates alone with helix $\alpha 9$ (Fig. 2E). When PKM2 adopts the R-state conformation, oxalate is recognized in the active site by residues R294, G295 and D296 of $\alpha 8$ on monomer A. The three residues are stabilized by residue $\mathrm{R} 342$ of $\alpha 11$ on monomer $\mathrm{C}$ through forming two intermolecular hydrogen bonds. In the process of an R- to T-state transition, helices a10/a11/a13 on monomer A move away from a10/a11/a13 on monomer $C$, whereas helices a14/a15/a16 on monomer A move closer to $\alpha 14 / \alpha 15 / \alpha 16$ on monomer $C$. The 
Table 1. Crystallographic data and structure refinement statistics

\begin{tabular}{|c|c|c|c|c|c|}
\hline Protein & $\mathrm{PKM}^{\mathrm{Y} 105 \mathrm{E}}$ & $\mathrm{PKM} 2^{\mathrm{K} 305 \mathrm{Q}}$ & $\mathrm{PKM}^{\mathrm{R} 399 \mathrm{E}}$ & $\mathrm{PKM}^{\mathrm{K} 422 \mathrm{R}}$ & $\mathrm{PKM} 2^{\mathrm{K} 422 \mathrm{R} \_\mathrm{FBP}}$ \\
\hline \multicolumn{6}{|l|}{ Data collection } \\
\hline Wavelength $(\AA)$ & 0.97927 & 0.97927 & 0.97923 & 0.97923 & 0.97923 \\
\hline $\begin{array}{l}\text { Resolution } \\
\text { range }(\AA)\end{array}$ & $\begin{array}{l}50-3.2 \\
(3.31-3.20)^{a}\end{array}$ & $\begin{array}{l}50-2.3 \\
(2.38-2.30)\end{array}$ & $\begin{array}{l}50-2.4 \\
(2.49-2.40)\end{array}$ & $\begin{array}{l}50-2.3 \\
(2.38-2.30)\end{array}$ & $\begin{array}{l}50-2.6 \\
(2.69-2.60)\end{array}$ \\
\hline Space group & $P 31$ & $P 1211$ & $P 1211$ & $P 1211$ & $P 1211$ \\
\hline Unit cell $\left(\AA,{ }^{\circ}\right)$ & $\begin{array}{c}124.5,124.5,257.0 \\
90.0,90.0,120.0\end{array}$ & $\begin{array}{r}94.8,117.4,110.3 \\
90.0,113.2,90.0\end{array}$ & $\begin{array}{c}95.6,71.2 \\
170.3,90 \\
104.3,90\end{array}$ & $\begin{array}{r}97.9,70.9,169.5 \\
90.0,100.2,90.0\end{array}$ & $\begin{array}{r}81.7,152.6,97.7 \\
90.0,104.2,90.0\end{array}$ \\
\hline Total reflections & 610595 & 610809 & 513532 & 581223 & 411179 \\
\hline $\begin{array}{l}\text { Unique } \\
\text { reflections }\end{array}$ & 72016 & 98003 & 87781 & 98424 & 70893 \\
\hline Multiplicity & $8.5(8.3)$ & $6.2(6.3)$ & $5.8(5.8)$ & $5.9(5.9)$ & $5.6(5.8)$ \\
\hline $\begin{array}{l}\text { Completeness } \\
(\%)\end{array}$ & $98.72(88.38)$ & 99.52 (95.59) & $98.30(84.27)$ & $96.72(89.08)$ & $98.9(100)$ \\
\hline Mean I/sigma $(I)$ & $5.56(2.87)$ & $11.35(3.33)$ & $7.34(3.91)$ & $7.47(2.93)$ & $35.0(2.68)$ \\
\hline$R$-sym & $0.192(0.768)$ & $0.098(0.552)$ & $0.166(0.83)$ & $0.137(0.676)$ & $0.084(0.854)$ \\
\hline \multicolumn{6}{|c|}{ Structure refinement } \\
\hline$R$-factor & 0.2202 & 0.2131 & 0.2101 & 0.1974 & 0.2759 \\
\hline$R$-free ${ }^{b}$ & 0.2652 & 0.2601 & 0.2562 & 0.2397 & 0.3036 \\
\hline RMS (bonds) & 0.010 & 0.002 & 0.002 & 0.002 & 0.005 \\
\hline RMS (angles) & 1.39 & 0.63 & 0.57 & 0.60 & 0.87 \\
\hline $\begin{array}{l}\text { Average } \\
\text { B-factor }\end{array}$ & 40.90 & 25.20 & 32.30 & 37.80 & 89.70 \\
\hline \multicolumn{6}{|c|}{ Ramachandran plot statistics } \\
\hline $\begin{array}{r}\text { Most favored } \\
\text { regions (\%) }\end{array}$ & 84.2 & 91.8 & 92.2 & 90.7 & 93.5 \\
\hline $\begin{array}{l}\text { Allowed regions } \\
(\%)\end{array}$ & 14.1 & 7.9 & 7.2 & 8.6 & 5.8 \\
\hline $\begin{array}{l}\text { Generously } \\
\text { allowed } \\
\text { regions (\%) }\end{array}$ & 1.4 & 0.3 & 0.3 & 0.4 & 0.4 \\
\hline $\begin{array}{l}\text { Disallowed } \\
\text { regions (\%) }\end{array}$ & 0.3 & 0 & 0.3 & 0.3 & 0.3 \\
\hline
\end{tabular}

a The values for the data in the highest resolution shell are shown in parentheses.

b $R$ free $=\sum$ Test ||$F o b s|-| F_{\text {calc }}|| \sum$ Test $\mid$ Fobs $\mid$, where "Test" is a test set of about $5 \%$ of the total reflections randomly chosen and set aside prior to refinement for the structure.

conformational change leads to a flip of the side chain on residue $\mathrm{R} 342$ on monomer $\mathrm{C}$, which stabilizes residues R294/G295/D296 on monomer A in the R-state conformation. As a result, PKM2 forms an inactive T-state tetramer, in which oxalate is not well recognized in the active site (Fig. 2F). The mutation R342 W of PKM2 abolished its enzymatic activity, further supporting its significance for catalysis (Fig. S1G). Consistent with above observations, the distances between residues K311 (in a9) on monomer A and $\mathrm{K} 311$ (in a9) on monomer $\mathrm{C}$ are approximate $18.0 \AA$ in the two states (Table S2). In contrast, the distance between residues E373 (in a13) on monomer A and E373 (in a13) on monomer $C$ is $\sim 37.8 \AA$ in the T-state and $\sim 31.1 \AA$ in the $\mathrm{R}$-state conformation; the distance between residues V414 (in a15) on monomer A and V414 (in a15) on monomer $C$ is $\sim 36.3 \AA$ in the T-state and $\sim 40.5 \AA$ in the R-state conformation.

In support of the above conformational changes, the transition of PKM2 from the R-state to the T-state, the distance between $\beta 20$ on monomer $A$ and $\beta 20$ on monomer $C$ along with the $\mathrm{C}-\mathrm{C}$ interface decreases from $\sim 59.6 \AA$ to $\sim 53.6 \AA$, whereas the distance between $\alpha 9$ on monomer $A$ and $\alpha 9$ on monomer $B$ along with the $A-A^{\prime}$ interface increases from $\sim 43.4 \AA$ to $\sim 51.6 \AA$ (Table S2). Regulation of 
A

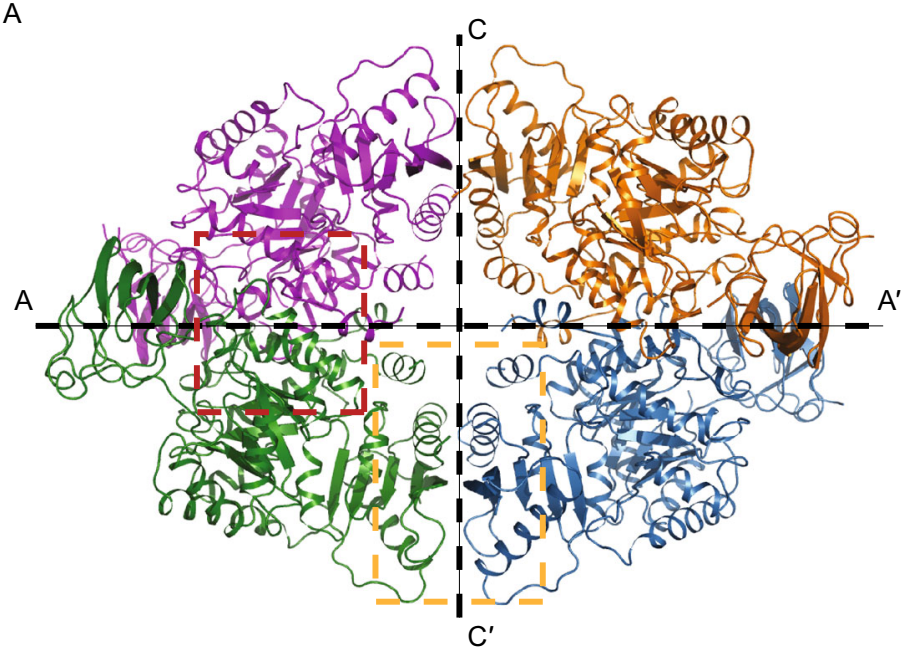

B

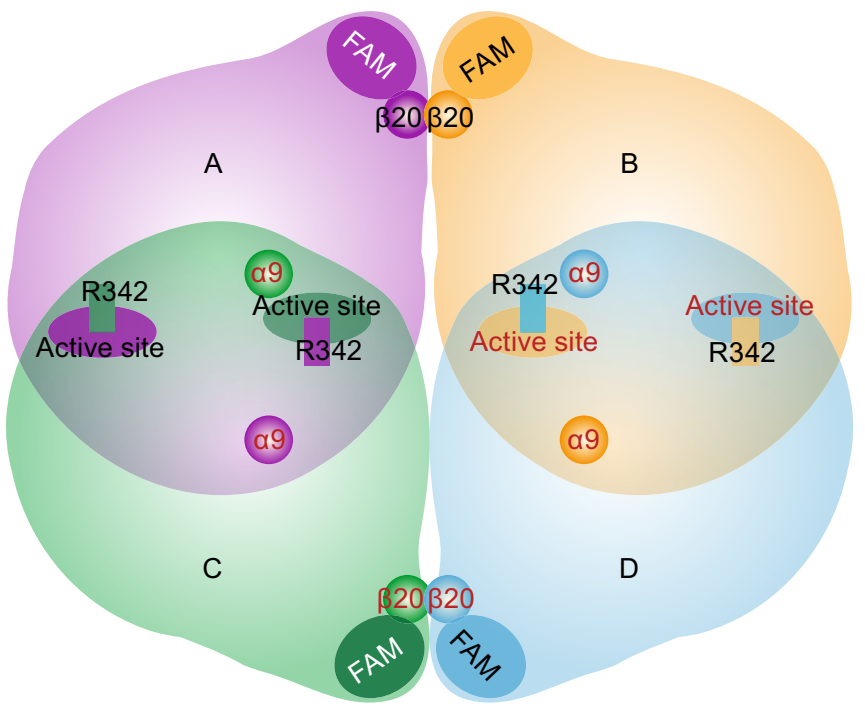

C

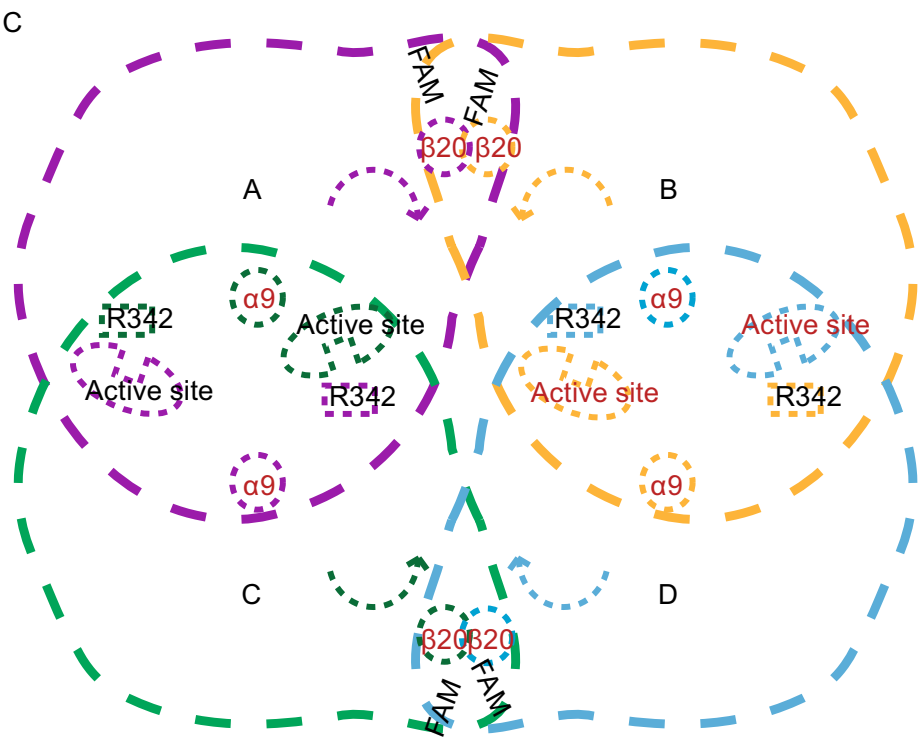

D
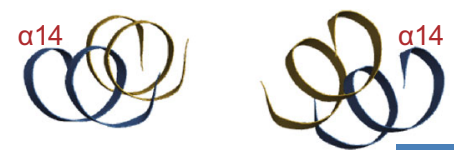

$\mathrm{PKM} 2^{\mathrm{K} 422 \mathrm{R}}$



E


F




Figure 2. Crystal structure of human PKM2 and a "seesaw" model for conformational transitions of PKM2 tetramer. (A) Ribbon representation of the human PKM2 structure $\left(\mathrm{PKM}^{\mathrm{K} 422 \mathrm{R}}\right.$ ) with $\mathrm{A}-\mathrm{A}^{\prime}$ (red box) and $\mathrm{C}^{-\mathrm{C}^{\prime}}$ (yellow box) interfaces indicated as dashed lines. Four monomers are shown in different colors. (B and C) A "seesaw" model for the conformational transitions between the R-state (B) and T-state (C) conformations of the PKM2 tetramer. Critical elements for the conformational changes are indicated. Dashed arrows indicate the directions for the rotation of each monomer from the R- $(B)$ to the T- (C) state. The monomers are indicated as monomer $A$ to $D$ for simplicity in the following description. ( $D$ and E) A close-up view for the structural comparison of the PKM2 structure in the R- and T-state conformations on the C-C' (D) or A-A' (E) interface. PKM2 in R- and T-state conformations are colored in yellow and blue, respectively. (F) A structural comparison of the active site of PKM2 in R- and T-state tetramers. PKM2 in R- and T-state conformations are colored in yellow and blue, respectively.

intermolecular interactions on either the A-A' (PEP binding site) or C-C' ( $\alpha 14 / \alpha 15$ or FAM/ 18 ) interface will lead to an R-/T-state transition of PKM2 and affect its enzymatic activity. For example, association of PEP or its analog oxalate of PKM2 induces an R-state conformation through enhancing intermolecular interactions on the $A-A^{\prime}$ interface. In addition, FBP recruits FAM back to the FBP binding pocket of PKM2 and disrupts the intermolecular interactions on the $\mathrm{C}$ - $\mathrm{C}^{\prime}$ interface to form an R-state conformation (Fig. 2B and 2C).

\section{PKM2 ${ }^{\text {K305Q }}$ disrupts tetramer formation and impairs} enzymatic activity

$\mathrm{PKM} 2^{\mathrm{K} 305 \mathrm{Q}}$ adopts a similar R-state conformation to that of PKM2 ${ }^{\text {Oxalate }}$ with a rmsd of $0.48 \AA$ for 1750 aligned $\mathrm{C} \alpha$ atoms (Fig. 3). The existence of malonate in the condition of crystallization may lead to an active R-state conformation of $\mathrm{PKM} 2^{\mathrm{K} 305 \mathrm{Q}}$ (Fig. S2B). No significant conformational change was observed for the two compared structures (Fig. S2A). However, differences exist between PKM2 ${ }^{\mathrm{K} 305 \mathrm{Q}}$ and $\mathrm{PKM} 2{ }^{\text {Oxalate }}$. In the PKM2 ${ }^{\text {Oxalate }}$ structure, two hydrogen bonds are formed between (1) residues K305 (on helix 99 of monomer C) and E384 (on helix a13) on monomer A and (2) K305 and I35 (close to helix a1) on monomer A (Fig. 3). The hydrogen bonds facilitate the intermolecular interactions on the A-A' interface. No such contact was observed in $\mathrm{PKM} 2^{\mathrm{K} 305 \mathrm{Q}}$ structure.

The FBP binding pocket on the $\mathrm{C}^{-\mathrm{C}^{\prime}}$ interface of $\mathrm{PKM} 2^{\mathrm{K} 305 \mathrm{Q}}$ are structurally similar to that of $\mathrm{PKM} 2^{\text {Oxalate }}$. Consistently, the enzymatic activity of PKM2 ${ }^{\mathrm{K} 305 \mathrm{Q}}$ could be slightly elevated by FBP (Fig. 1C). Gel-filtration and thermalshift assays demonstrated that FBP retains its ability to stabilize PKM2 ${ }^{\mathrm{K} 305 \mathrm{Q}}$ and promote its dimer formation (Figs. 1D, 1E and S1E). Altogether, mutation $\mathrm{K} 305 \mathrm{Q}$ or acetylation of residue K305 inhibits PKM2 activity through hindering the tetramer formation on $\mathrm{A}-\mathrm{A}^{\prime}$ interface.
$\mathrm{PKM}^{\mathrm{K} 422 \mathrm{R}}$ forms a tetramer in the T-state in the absence of FBP and the R-state in the presence of FBP

Different from $\mathrm{PKM} 2^{\mathrm{O} x a l a t e}$ or $\mathrm{PKM} 2^{\mathrm{K} 305 \mathrm{Q}}, \mathrm{PKM} 2^{\mathrm{K} 422 \mathrm{R}}$ adopts a T-state conformation in the absence of FBP and an R-state conformation in the presence of FBP (Fig. S2A). In the $\mathrm{PKM} 2^{\text {Oxalate }}$ structure, two hydrogen bonds are formed between residue K422 (on monomer $\mathrm{C}$ ) and residues $\mathrm{P} 403$ and Y444 (on monomer D), whereas FAM is flexible and invisible (Fig. 3). In the PKM2 ${ }^{\mathrm{K} 422 \mathrm{R}}$ structure, residue K422R on monomer $\mathrm{C}$ forms two hydrogen bonds with residue $\mathrm{E} 418$ on monomer $\mathrm{D}$. Intermolecular interactions are mediated by hydrogen bonds between residue D487 on a18 of monomer C and W515 and R516 on FAM of monomer D, and hydrophobic interactions between residues W482, W515 and P517 on both monomer $\mathrm{C}$ and monomer D. The FBP association stabilizes the FAM and disrupts the interactions between FAM on monomer $C$ and $\alpha 18$ on monomer $D$, therefore allowing the tetramer to adopt an R-state conformation (Fig. 3).

The $\mathrm{PKM} 2^{\mathrm{K} 422 \mathrm{R}}$ tetramer is structurally similar to the $\mathrm{PKM}^{\text {Phe }}$ tetramer (PDB: 4FXJ) with a rmsd of $0.41 \AA$ for 1720 aligned $\mathrm{Ca}$ atoms (Fig. 4A). Compared to PKM2 ${ }^{\text {Phe }}$, $\mathrm{PKM} 2^{\mathrm{K} 422 \mathrm{R}}$ has two additional hydrogen bonds between residue $\mathrm{K} 422 \mathrm{R}$ on $\alpha 15$ on monomer $\mathrm{C}$ and $\mathrm{E} 418$ on $\alpha 15$ on monomer $D$, which may lead to the dissociation of helices $\alpha 14$ on monomer $C$ and helices $\alpha 15$ on monomer $D$, and therefore strengthen intermolecular interactions ( $\alpha 15$ on monomer $\mathrm{C}$ and $\alpha 15$ on monomer $\mathrm{D}$ ) on the $\mathrm{C}-\mathrm{C}^{\prime}$ interface to facilitate the T-state tetramer formation. The strong intermolecular interactions on the $\mathrm{C}^{-\mathrm{C}^{\prime}}$ interface in $\mathrm{PKM} 2^{\mathrm{K} 422 \mathrm{R}}$ may be difficult to disrupt by PEP to form an R-state conformation. The above structural analyses support the observations that $\mathrm{PKM} 2^{\mathrm{K} 422 \mathrm{R}}$ forms a less active tetramer in solution (compared to $\mathrm{PKM} 2^{\mathrm{WT}}$ ) but is stable in the thermalshift assay (Figs. 1C, 1D and S1E).

Although PKM2 ${ }^{\mathrm{K} 422 \mathrm{R}}$ tends not to adopt a PEP-induced $R$-state conformation because of strong intermolecular interactions on the $\mathrm{C}-\mathrm{C}^{\prime}$ interface (compared to $\mathrm{PKM} 2^{\text {Oxalate }}$ ), association of FBP did favor the R-state tetramer formation. Our structure of $\mathrm{PKM} 2^{\mathrm{K} 422 \mathrm{R}} \mathrm{FBP}$ offers direct evidence for the R-state formation: the $\mathrm{PKM} 2^{\mathrm{K} 422 \mathrm{R}}$ tetramer adopts a similar fold to that of PKM2 ${ }^{\mathrm{WT}}$ bound to FBP (PDB: 4B2D) (Fig. 4B). In support of the above observations, PKM2 $2^{\mathrm{K} 422 \mathrm{R}}$ showed pyruvate kinase activity comparable to that of $\mathrm{PKM}^{\mathrm{WT}}$ in the presence of $\mathrm{FBP}$, but significantly decreased activity and enhanced cooperativity in the absence of FBP (Fig. 1C and Table S1).

$\mathrm{PKM}^{\mathrm{R} 399 \mathrm{E}}$ tends to inhibit the formation of R-state tetramer

$\mathrm{PKM}^{\mathrm{R} 399 \mathrm{E}}$ also adopts the T-state conformation (Fig. S2A). No significant difference existed between the structures of $\mathrm{PKM}^{\mathrm{R} 399 \mathrm{E}}$ and PKM2 ${ }^{\mathrm{Phe}}$ (Fig. 4A). Residue R399 of $\alpha 14$ on 

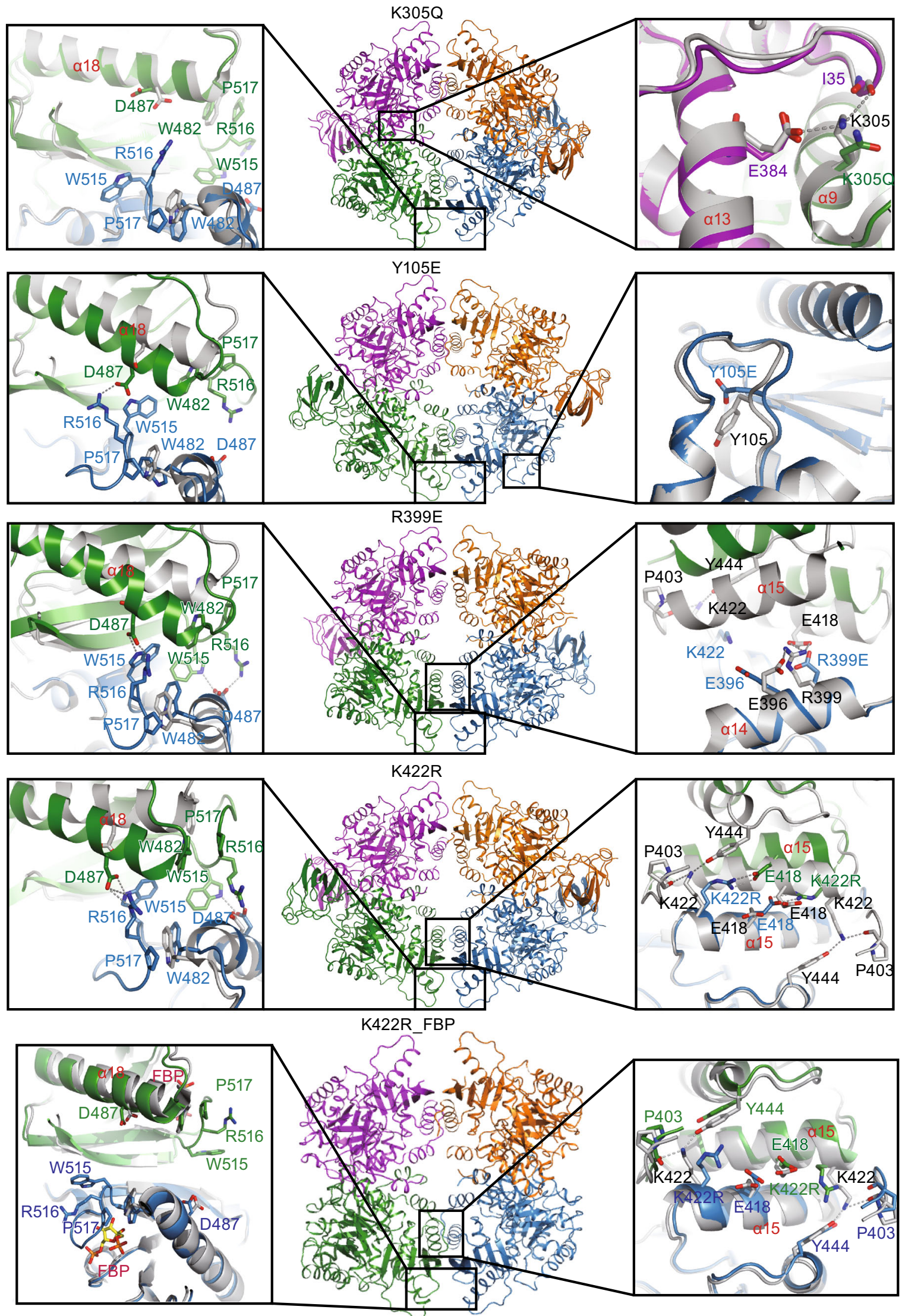
Figure 3. Structural comparisons of PKM2 mutants and PKM2 ${ }^{\text {Oxalate }}$ (in the R-state). Close-up views of PKM2 mutants with critical residues indicated as stick representations. $\mathrm{PKM} 2^{\mathrm{O} x a l a t e}$ (in the R-state) is colored in gray.

monomer $\mathrm{C}$ forms hydrogen bonds with residues $\mathrm{E} 396$ of a 14 and E418 of $\alpha 15$ on monomer D on the C-C' interface of the PKM2 ${ }^{\text {WT }}$ structure. However, residue R399E on monomer $C$ may not form the same hydrogen bonds in PKM2 ${ }^{R 399 E}$ even in the R-state (in the presence of PEP or FBP) because the three negatively charged residues may repel one another (Fig. 3). The existence of R399E in PKM2 will inhibit the formation of the R-state conformation induced by FBP or $P E P$, and lead to a decrease of enzymatic activity (compared to $\mathrm{PKM} 2^{\mathrm{WT}}$ ). Consistently, in the presence of FBP, $\mathrm{PKM}^{\mathrm{R} 399 \mathrm{E}}$ is less active and has less of a tendency to form a tetramer compared to $\mathrm{PKM} 2^{\mathrm{WT}}$ (Fig. $1 \mathrm{C}$ and $1 \mathrm{E}$ ). In contrast, no such significant difference in the extent of tetramer formation was observed in the absence of FBP.

PKM2 ${ }^{\mathrm{Y} 105 E}$ inhibits FBP association and formation of the FBP-induced R-state of PKM2

A previous study indicated that phosphorylation of residue Y105 inhibits FBP association and tetramer formation of PKM2, and thus decreases its enzymatic activity (Hitosugi et al., 2009). In the crystal structure, PKM2 ${ }^{\mathrm{Y} 105 \mathrm{E}}$ binds to proline, which was derived from a crystallization condition and might have facilitated a T-state conformation (as did phenylalanine) (Fig. S2D). No significant conformational change around residue $\mathrm{Y} 105 \mathrm{E}$ was observed between structures of $\mathrm{PKM} 2^{\mathrm{Y} 105 \mathrm{E}}$ and $\mathrm{PKM} 2^{\text {Oxalate }}$ (Fig. 3). The crystal structure could not reveal the mechanism for the inhibition of FBP association. However, it is possible that phosphorylation of $\mathrm{Y} 105$ functions during the dynamic transition, which could not be observed in crystal structure. In support of this conjecture, $\mathrm{PKM} 2^{\mathrm{Y} 105 \mathrm{E}}$ has similar enzymatic activity and tetramer formation (compared to $\mathrm{PKM} 2^{\mathrm{WT}}$ ) in the absence of FBP (Fig. 1C and 1D). The addition of oxalate leads to an increase in tetramer formation to a comparable extent for PKM2 ${ }^{\mathrm{Y} 105 \mathrm{E}}$ and $\mathrm{PKM} 2^{\mathrm{WT}}$ (Fig. S1F). In contrast, the addition of FBP results in significantly less of an increase in the extent of tetramer formation for PKM2 ${ }^{\mathrm{Y} 105 \mathrm{E}}$ (compared to $\mathrm{PKM} 2{ }^{\mathrm{WT}}$ ) (Fig. 1E). Taken together, the mutation $\mathrm{Y} 105 \mathrm{E}$, or phosphorylation of residue $\mathrm{Y} 105$, inhibits FBP association and prevents the FBP-induced R-state formation of PKM2.

\section{DISCUSSION}

\section{A working model for the dynamic regulation of PKM2}

Based on above analyses, here we propose a working model for the dynamic regulation of PKM2 (Fig. 5). PKM2 exists in a mixed population of monomer, dimer and tetramer, and prefers a T-state conformation if the tetramer forms in the absence of FBP. Upon FBP association, PKM2 undergoes a change from the T-state to the R-state conformation, which favors the recognition of PEP in the active site and enhances its pyruvate kinase activity. Regulation of the intermolecular interactions on the $A-A^{\prime}$ or $C-C^{\prime}$ interface plays an important role in conformational changes of PKM2. A "Rock and Lock" model has been proposed based on structural studies of pyruvate kinase from L. Mexicana (Morgan et al., 2010). However, the model was not sufficient to reveal the mechanism for dynamic regulation of human PKM2. In this work, a "seesaw" model was proposed based on the comparison of human PKM2 structures in T- and R-state tetramers (Fig. $2 \mathrm{~B}$ and $2 \mathrm{C}$ ). Our biochemical and structural studies revealed the mechanisms for dynamic regulation of the pyruvate kinase activity of PKM2 by inhibitor/activator, post-translational modifications and a patientderived mutation (Fig. 5). The two layers of regulation provide precise control of pyruvate kinase activity of PKM2 in various biological and pathological conditions.

Post-translational modifications (such as acetylation and phosphorylation) and the observed patient-derived mutation regulate PKM2 pyruvate kinase activity through modulating tetramer formation or the transition between T- and R-state conformations (Fig. 5). PKM2 with an acetylated K305 mainly forms a monomer in solution and forms a dimer in the presence of FBP because the protein loses the intermolecular interactions on the $A-A^{\prime}$ interface. The lack of tetramer formation leads to a significant decrease in the enzymatic activity. Phosphorylation of residue Y105 inhibits FBP association and prevents the FBP-induced R-state formation of PKM2, but experiences minor effects in the absence of FBP. Acetylation of residue K433 was reported to decrease PKM2 activity through inhibiting FBP association (Lv et al., 2013). $P K M 2^{R 399 E}$ tends to inhibit the formation of R-state tetramer (R399E on monomer A repels E396 on monomer $A$ and $\mathrm{E} 418$ on monomer $\mathrm{B}$ on the $\mathrm{C}-\mathrm{C}^{\prime}$ interface) and leads to a decrease of activity even in the presence of FBP. Interestingly, $\mathrm{PKM} 2^{\mathrm{K} 422 \mathrm{R}}$ tends not to form the R-state conformation in the absence of FBP because the hydrogen bonds between residues $\mathrm{K} 422 \mathrm{R}$ on monomer $\mathrm{A}$ and $\mathrm{E} 418$ on monomer $B$ on the $C-C^{\prime}$ interface favor a stable T-state tetramer formation. Moreover, FBP could induce the re-formation of the R-state tetramer of PKM2 ${ }^{\mathrm{K} 422 \mathrm{R}}$. Thus, $\mathrm{PKM} 2^{\mathrm{K} 422 \mathrm{R}}$ shows low activity and high cooperativity in the absence of FBP but comparable activity to that of $\mathrm{PKM} 2^{\mathrm{WT}}$ in the presence of FBP. Notably, the cellular concentration of PEP ranges from $4 \mu \mathrm{mol} / \mathrm{L}$ to $140 \mu \mathrm{mol} / \mathrm{L}$ in human and rat tissues (Liebermeister, 2005). Under such conditions, PKM2 ${ }^{\mathrm{K} 305 \mathrm{Q}}$ and $\mathrm{PKM} 2^{\mathrm{K} 422 \mathrm{R}}$ show a significant decrease in pyruvate kinase activity (compared to PKM2 ${ }^{\mathrm{WT}}$ ). Consistently, acetylation of $\mathrm{K} 305$ on PKM2 changes metabolic intermediates and promotes cell proliferation and tumor growth, and the K422R mutation of PKM2 enhances HeLa cell proliferation (Gupta et al., 2010; Lv et al., 2011).

The pyruvate kinase activity of PKM2 could be regulated by small molecules. FBP could recruit the FAM to the FBP binding pocket on the $C-C^{\prime}$ interface and facilitate R-state 
A


B


Figure 4. Structural comparisons of $\mathrm{PKM} 2$ mutants with $\mathrm{PKM} 2^{\mathrm{Phe}}$ (in T-state) and $\mathrm{PKM} 2^{\mathrm{K} 422 \mathrm{R} \_\mathrm{FBP}}$ with $\mathrm{PKM} 2$ wild type bound with FBP. (A) Close-up views of PKM2 mutants with critical residues indicated as stick representation. PKM2 ${ }^{\mathrm{Phe}}$ (in T-state) is colored in gray. (B) Close-up views of $\mathrm{PKM} 2^{\mathrm{K} 422 \mathrm{R}}{ }^{\mathrm{FBP}}$ with critical residues indicated as stick representation. $\mathrm{PKM} 2^{\mathrm{FBP}}$ (in $\mathrm{R}$-state, PDB: 4B2D) is colored in gray. 


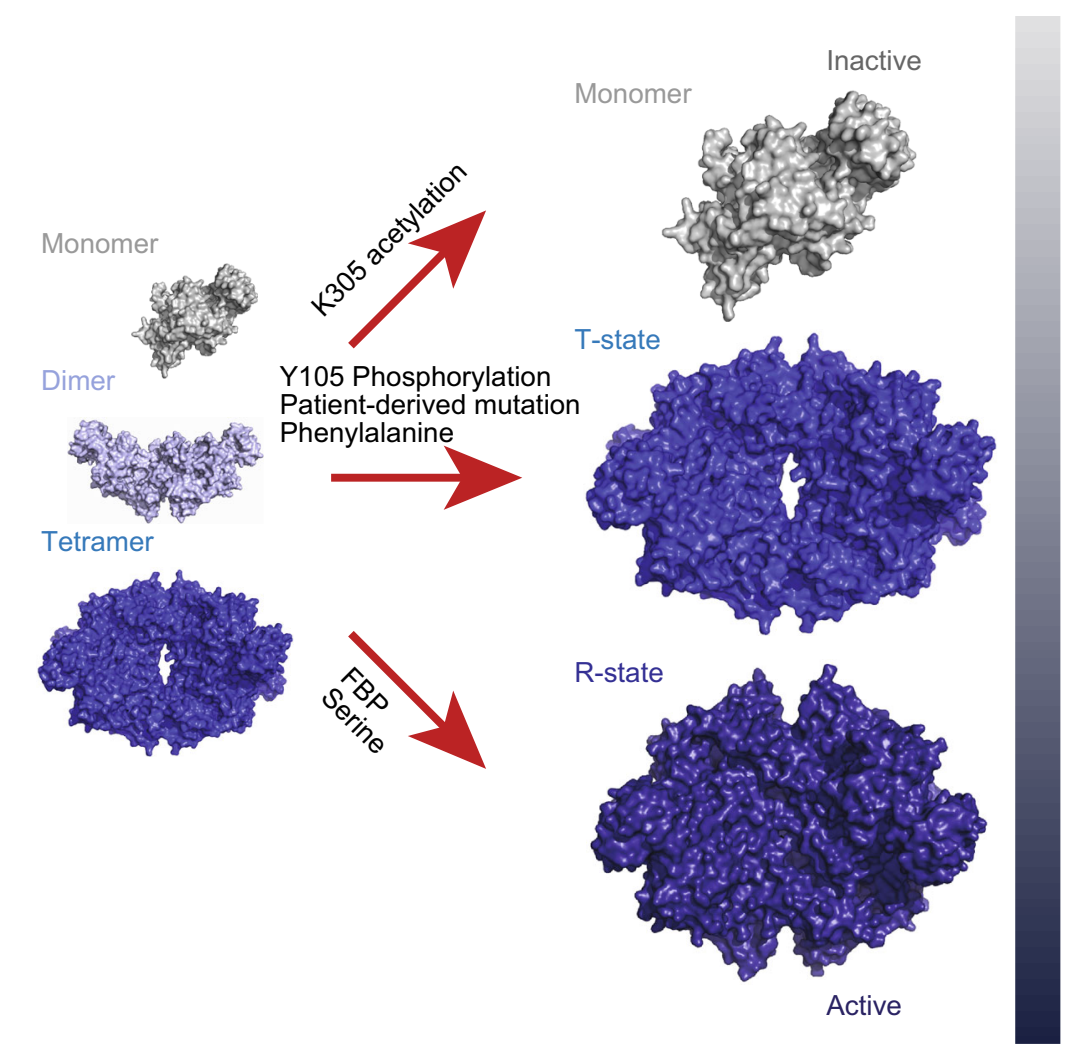

Figure 5. Regulation of PKM2 activity by inhibitor/activators, post-translational modifications and a patient-derived mutation. PKM2 exists in a mixed population of monomer, dimer and tetramer. Acetylation of residue K305 disrupts the tetramer formation. Phosphorylation of residue $\mathrm{Y} 105$, the patient derived mutation K422R and phenylalanine association induce an inactive T-state tetramer formation, whereas FBP and serine promote its active R-state formation. The level of pyruvate kinase activity of PKM2 is indicated by a colored bar, in which gray represents low activity and deep blue represents high activity.

tetramer formation of PKM2. PEP, or its analog (such as oxalate, malonate), associates with $\mathrm{PKM} 2$ on the $\mathrm{A}-\mathrm{A}^{\prime}$ interface and allosterically induces R-state tetramer formation. Another PKM2 activator, TEPP-46, enhances the intermolecular interactions on the A-A' interface and favors an R-state conformation (Anastasiou et al., 2012). The crystal structure of PKM2 bound to phenylalanine demonstrates that the amino acid could lock PKM2 in an inactive T-state (Morgan et al., 2013), while the crystal structure of PKM2 bound to serine demonstrates that the amino acid could also allosterically activate PKM2 (Chaneton et al., 2012). SAICAR is a metabolite abundant in proliferating cells and stimulates both pyruvate and protein kinase activities of PKM2 (Keller et al., 2012; Keller et al., 2014). PKM2 ${ }^{\text {Q393K }}$ (Q393 is located on helix a14) is a SAICAR-insensitive mutant, suggesting that the compound might regulate PKM2 through both $\mathrm{A}-\mathrm{A}^{\prime}$ and $\mathrm{C}-\mathrm{C}^{\prime}$ interfaces.

PKM2 has long been known to have pyruvate kinase activity. However, several studies recently indicated that PKM2 also possesses protein kinase activity, which plays an important role in tumorigenesis. For example, EGFR- activated ERK2 promotes nuclear translocation and protein kinase activity of PKM2 (Yang et al., 2012a; Yang et al., 2012b). SAICAR directly interacts with PKM2 and promotes its protein kinase activity (Keller et al., 2014). In addition, association of tyrosine phosphorylated peptide, acetylation of residue K433 or the mutation of R399E of PKM2 promotes its protein kinase activity and nuclear localization, which are correlated with predominant dimer formation (Gao et al., 2012; Gao et al., 2013; Lv et al., 2013). Interestingly, these dimers are formed with the loss of intermolecular interaction on the $\mathrm{C}-\mathrm{C}^{\prime}$ interface, suggesting that regulation on this interface may be responsible for the switch from pyruvate kinase activity to protein kinase activity of PKM2. Further structural and biochemical studies may reveal the mechanisms for the switch of substrate specificity of PKM2.

In summary, our study provides structural insight into dynamic regulation of PKM2 by post-translational modifications and a patient-derived mutation. The "seesaw" model reveals the mechanism for the transition between $\mathrm{T}$ - and R-state conformations and provides a platform for further investigation of other modifications/mutations of PKM2. 


\section{ACCESSION NUMBERS}

The atomic coordinates of the $\mathrm{PKM} 2^{\mathrm{Y} 105 \mathrm{E}}, \mathrm{PKM} 2^{\mathrm{K} 305 \mathrm{Q}}$, $\mathrm{PKM} 2^{\mathrm{R} 399 \mathrm{E}}, \mathrm{PKM} 2^{\mathrm{K} 422 \mathrm{R}}$, and PKM2 ${ }^{\mathrm{K} 422 \mathrm{R} \_\mathrm{FBP}}$ have been deposited in the Protein Data Bank with the PDB codes 4QG6, 4QG8, 4QG9, 4QGC, and 4RPP, respectively.

\section{MATERIALS AND METHODS}

\section{Protein crystallization}

The ORF of human PKM2 was kindly gifted from Kun-liang Guan's lab at Fudan University. Procedures for mutagenesis and protein purification can be found in the Supplemental Materials. The crystals for PKM2 mutants were obtained at $18^{\circ} \mathrm{C}$ by the hanging-drop, vapordiffusion method by mixing $1 \mu \mathrm{L}$ protein solution $(15-20 \mathrm{mg} / \mathrm{mL}$ ) with 1 $\mu \mathrm{L}$ reservoir solution containing $0.2 \mathrm{~mol} / \mathrm{L}$ malonate $\mathrm{pH} 5.0,20 \%$ PEG3350 (for PKM2 ${ }^{\mathrm{K} 305 \mathrm{Q}}$ ); $0.2 \mathrm{~mol} / \mathrm{L}$ L-proline, $0.1 \mathrm{~mol} / \mathrm{L} \mathrm{HEPES} \mathrm{pH}$ 7.5, 24\% PEG1500 (for PKM2 ${ }^{\mathrm{Y} 105 \mathrm{E}}$ ); $0.2 \mathrm{~mol} / \mathrm{L} \mathrm{CaAc}_{2}, 20 \%$ PEG3350 (for PKM2 ${ }^{\mathrm{R} 399 \mathrm{E}}$ ); $0.2 \mathrm{~mol} / \mathrm{L} \mathrm{K}_{2} \mathrm{SO}_{4}, 20 \% \mathrm{PEG} 3350$ (for PKM2 ${ }^{\mathrm{K} 422 \mathrm{R}}$ ); and $0.1 \mathrm{~mol} / \mathrm{L}$ sodium chloride, $0.1 \mathrm{~mol} / \mathrm{L}$ BIS-TRIS propane $\mathrm{pH} 9.0$, 25\% PEG 1500 for (PKM2 ${ }^{\text {K422R_FBP }}$ ).

Data collection and structure determination

Crystals were mounted on nylon loops and flash-cooled in a cold nitrogen stream at $100 \mathrm{~K}$. The data sets were collected at a single wavelength at the Shanghai Synchrotron Radiation Facility (SSRF) in China on beamline BL17U. Data were indexed, integrated and scaled using the HKL2000 program (Otwinowski and Minor 1997). The structures of PKM2 mutants were determined by molecular replacement using the monomer of PKM2 wild-type structure (3BJT. pdb) as a searching model (Christofk et al., 2008b). Details about structure determination and refinement can be found in Supplemental Materials.

\section{Measurement of pyruvate kinase activity}

A lactate dehydrogenase ( $\mathrm{LDH}$ )-coupled pyruvate kinase activity assay was performed to measure the activity of wild-type PKM2 and mutants of PKM2 as previously described (Morgan et al., 2013). Because the reaction was sigmoidal, the reaction rates of PKM2 (in the absence of FBP) were calculated according to the Allosteric Sigmoidal Equation. For the reaction rates of PKM2 in the presence of FBP, we performed the calculation according to Michaelis-Menten Equation. Details for the pyruvate kinase assay can be found in Supplemental Materials.

\section{Thermal shift assay and gel filtration assay}

The thermal-shift assay and the gel-filtration (Superdex 200, GE healthcare, $10 / 300 \mathrm{GL}$ ) assay were performed to examine the thermal stability and tetramer formation of PKM2 proteins. Detailed descriptions can be found in Supplemental Materials.

\section{ACKNOWLEDGMENTS}

We thank staff members of beamline BL17U at SSRF (Shanghai Synchrotron Radiation Facility, China) for their assistance in data collection, and staff members of Biomedical Core Facility, Fudan University for their help on biochemical analyses. This work was supported by grants from the National Natural Science Foundation of China (Grant Nos. 31270779 and 31425008), the Basic Research Project of Shanghai Science and Technology Commission (12JC1402700), the Program of Shanghai Subject Chief Scientist (14XD1400500), and "ShuGuang" project (11SG06) supported by Shanghai Municipal Education Commission and Shanghai Education Development Foundation.

\section{ABBREVIATIONS}

ADP, adenosine di-phosphate; ATP, adenosine tri-phosphate; FBP, fructose 1,6-bisphosphate; PEP, phosphoenolpyruvate; PKM2, pyruvate kinase isoform M2.

\section{COMPLIANCE WITH ETHICS GUIDELINES}

Ping Wang, Chang Sun, Tingting Zhu and Yanhui Xu declare that they have no conflict of interest.

This article does not contain any studies with human or animal subjects performed by the any of the authors.

\section{OPEN ACCESS}

This article is distributed under the terms of the Creative Commons Attribution License which permits any use, distribution, and reproduction in any medium, provided the original author(s) and the source are credited.

\section{REFERENCES}

Akhtar K, Gupta V, Koul A, Alam N, Bhat R, Bamezai RN (2009) Differential behavior of missense mutations in the intersubunit contact domain of the human pyruvate kinase M2 isozyme. J Biol Chem 284:11971-11981

Anastasiou D, Poulogiannis G, Asara JM, Boxer MB, Jiang JK, Shen M, Bellinger G, Sasaki AT, Locasale JW, Auld DS et al (2011) Inhibition of pyruvate kinase M2 by reactive oxygen species contributes to cellular antioxidant responses. Science 334:12781283

Anastasiou D, Yu Y, Israelsen WJ, Jiang JK, Boxer MB, Hong BS, Tempel W, Dimov S, Shen M, Jha A et al (2012) Pyruvate kinase M2 activators promote tetramer formation and suppress tumorigenesis. Nat Chem Biol 8:839-847

Anitha M, Kaur G, Baquer NZ, Bamezai R (2004) Dominant negative effect of novel mutations in pyruvate kinase-M2. DNA Cell Biol 23:442-449

Boxer MB, Jiang J, Vander Heiden MG, Shen M, Veith H, Cantley LC, Thomas CJ (2010) Identification of activators for the M2 isoform of human pyruvate kinase version 3. In: Probe reports from the NIH molecular libraries program. National Center for Biotechnology Information, Bethesda

Chaneton B, Gottlieb E (2012) Rocking cell metabolism: revised functions of the key glycolytic regulator PKM2 in cancer. Trends Biochem Sci 37:309-316

Chaneton B, Hillmann P, Zheng L, Martin AC, Maddocks OD, Chokkathukalam A, Coyle JE, Jankevics A, Holding FP, Vousden 
$\mathrm{KH}$ et al (2012) Serine is a natural ligand and allosteric activator of pyruvate kinase M2. Nature 491:458-462

Christofk HR, Vander Heiden MG, Harris MH, Ramanathan A, Gerszten RE, Wei R, Fleming MD, Schreiber SL, Cantley LC (2008a) The M2 splice isoform of pyruvate kinase is important for cancer metabolism and tumour growth. Nature 452:230-233

Christofk HR, Vander Heiden MG, Wu N, Asara JM, Cantley LC (2008b) Pyruvate kinase M2 is a phosphotyrosine-binding protein. Nature 452:181-186

Dombrauckas JD, Santarsiero BD, Mesecar AD (2005) Structural basis for tumor pyruvate kinase M2 allosteric regulation and catalysis. Biochemistry 44:9417-9429

Gao X, Wang H, Yang JJ, Liu X, Liu ZR (2012) Pyruvate kinase M2 regulates gene transcription by acting as a protein kinase. Mol Cell 45:598-609

Gao X, Wang H, Yang JJ, Chen J, Jie J, Li L, Zhang Y, Liu ZR (2013) Reciprocal regulation of protein kinase and pyruvate kinase activities of pyruvate kinase M2 by growth signals. J Biol Chem 288:15971-15979

Gupta V, Kalaiarasan P, Faheem M, Singh N, Iqbal MA, Bamezai RN (2010) Dominant negative mutations affect oligomerization of human pyruvate kinase $\mathrm{M} 2$ isozyme and promote cellular growth and polyploidy. J Biol Chem 285:16864-16873

Hitosugi T, Kang S, Vander Heiden MG, Chung TW, Elf S, Lythgoe K, Dong S, Lonial S, Wang X, Chen GZ et al (2009) Tyrosine phosphorylation inhibits PKM2 to promote the Warburg effect and tumor growth. Sci Signal 2:ra73

Iqbal MA, Siddiqui FA, Chaman N, Gupta V, Kumar B, Gopinath P, Bamezai RN (2014) Missense mutations in pyruvate kinase M2 promote cancer metabolism, oxidative endurance, anchorage independence and tumor growth in a dominant negative manner. J Biol Chem 289:8098-8105

Jiang JK, Boxer MB, Vander Heiden MG, Shen M, Skoumbourdis AP, Southall N, Veith H, Leister W, Austin CP, Park HW et al (2010) Evaluation of thieno[3,2-b]pyrrole[3,2-d]pyridazinones as activators of the tumor cell specific M2 isoform of pyruvate kinase. Bioorg Med Chem Lett 20:3387-3393

Keller KE, Tan IS, Lee YS (2012) SAICAR stimulates pyruvate kinase isoform M2 and promotes cancer cell survival in glucoselimited conditions. Science 338:1069-1072

Keller KE, Doctor ZM, Dwyer ZW, Lee Y-S (2014) SAICAR induces protein kinase activity of PKM2 that is necessary for sustained proliferative signaling of cancer cells. Mol Cell 53:700-709

Koppenol WH, Bounds PL, Dang CV (2011) Otto Warburg's contributions to current concepts of cancer metabolism. Nat Rev Cancer 11:325-337

Kung C, Hixon J, Choe S, Marks K, Gross S, Murphy E, DeLaBarre B, Cianchetta G, Sethumadhavan S, Wang X et al (2012) Small molecule activation of PKM2 in cancer cells induces serine auxotrophy. Chem Biol 19:1187-1198
Liebermeister W (2005) Predicting physiological concentrations of metabolites from their molecular structure. J Comput Biol 12:1307-1315

Luo W, Hu H, Chang R, Zhong J, Knabel M, O'Meally R, Cole RN, Pandey A, Semenza GL (2011) Pyruvate kinase M2 is a PHD3stimulated coactivator for hypoxia-inducible factor 1 . Cell 145:732-744

Lv L, Li D, Zhao D, Lin R, Chu Y, Zhang H, Zha Z, Liu Y, Li Z, Xu Y et al (2011) Acetylation targets the $M 2$ isoform of pyruvate kinase for degradation through chaperone-mediated autophagy and promotes tumor growth. Mol Cell 42:719-730

Lv L, Xu YP, Zhao D, Li FL, Wang W, Sasaki N, Jiang Y, Zhou X, Li TT, Guan KL et al (2013) Mitogenic and oncogenic stimulation of K433 acetylation promotes PKM2 protein kinase activity and nuclear localization. Mol Cell 52:340-352

Morgan HP, McNae IW, Nowicki MW, Hannaert V, Michels PA, Fothergill-Gilmore LA, Walkinshaw MD (2010) Allosteric mechanism of pyruvate kinase from Leishmania mexicana uses a rock and lock model. J Biol Chem 285:12892-12898

Morgan HP, O'Reilly FJ, Wear MA, O'Neill JR, Fothergill-Gilmore LA, Hupp T, Walkinshaw MD (2013) M2 pyruvate kinase provides a mechanism for nutrient sensing and regulation of cell proliferation. Proc Natl Acad Sci U S A 110:5881-5886

Noguchi T, Inoue H, Tanaka T (1986) The M1- and M2-type isozymes of rat pyruvate kinase are produced from the same gene by alternative RNA splicing. J Biol Chem 261:13807-13812

Noguchi T, Yamada K, Inoue H, Matsuda T, Tanaka T (1987) The Land R-type isozymes of rat pyruvate kinase are produced from a single gene by use of different promoters. J Biol Chem 262:14366-14371

Otwinowski Z, Minor W (1997) Processing of X-ray diffraction data collected in oscillation mode. Methods Enzymol 276:307-326

Vander Heiden MG, Cantley LC, Thompson CB (2009) Understanding the Warburg effect: the metabolic requirements of cell proliferation. Science 324:1029-1033

Warburg O (1956) On the origin of cancer cells. Science 123:309323

Wong N, Ojo D, Yan J, Tang D (2015) PKM2 contributes to cancer metabolism. Cancer Lett 356:184-191

Yang W, Lu Z (2013) Regulation and function of pyruvate kinase M2 in cancer. Cancer Lett 339:153-158

Yang W, Xia Y, Hawke D, Li X, Liang J, Xing D, Aldape K, Hunter T, Alfred Yung WK, Lu Z (2012a) PKM2 phosphorylates histone H3 and promotes gene transcription and tumorigenesis. Cell 150:685-696

Yang W, Zheng Y, Xia Y, Ji H, Chen X, Guo F, Lyssiotis CA, Aldape K, Cantley LC, Lu Z (2012b) ERK1/2-dependent phosphorylation and nuclear translocation of PKM2 promotes the Warburg effect. Nat Cell Biol 14:1295-1304 